\title{
Inhibitory Potency of Selected Therapeutic Bioactive Molecules of Standardized Terminalia arjuna (Roxb.) Extract on CYP3A4 and CYP2D6: Exploring Possible Herb-Drug Interactions.
}

\author{
Vijayakumar $\mathrm{TM}^{1^{*}}$, Ilango $\mathrm{K}^{2}$, Vasanth $\mathrm{K}^{2}$, Kasthuri Bai $\mathbf{N}^{2}$, Mohan Kumar $\mathbf{R}^{2}$ and Dubey $\mathbf{G P}^{3}$ \\ ${ }^{1}$ Department of Pharmacy Practice, SRM College of Pharmacy, SRM University, Kattankulathur, Tamil Nadu, India \\ ${ }^{2}$ Interdisciplinary Institute of Indian System of Medicine, SRM University, Kattankulathur, Tamil Nadu, India
}

${ }^{3}$ Department of Kriya Sharir, Institute of Medical Sciences (IMS), Banaras Hindu University, Varanasi, Uttar Pradesh, India

\begin{abstract}
A number of significant herbal drug interactions have their origins in the alteration of cytochrome P450 (CYP) activity by various phytochemicals. In the present study, inhibition potential of Terminalia arjuna extracts and its constituent gallic acid (GA) and ellagic acid (EA) to cause herb-drug interactions through rat liver cytochrome enzymes, CYP3A4, and CYP2D6 was evaluated, a rapid RP-HPLC method was developed for quantitative estimation of GA and EA in the extract. In vitro safety of the extract and active components were evaluated through CYP450 inhibition method using pooled rat microsomes and high throughput fluorometric assay with CYP3A4 and CYP2D6. The binding mode and the molecular interaction of GA and EA within the CYP3A4 and CYP2D6 active site were demonstrated using an in-silico docking studies. From CYP450-CO Complex assay, the inhibitory potential of $T$. arjuna standardized extract (31.02 \pm $2.24 \%$ ), was found to be less than positive control. In high throughput fluorometric assay, $T$. arjuna extracts exhibited higher $I_{50}$ values $(48.06 \pm 1.14-57.89 \pm 2.15 \mu \mathrm{g} / \mathrm{mL})$ compared to positive inhibitors and lower than GA $(66.54 \pm$ $1.04-83.84 \pm 1.06 \mu \mathrm{g} / \mathrm{mL}), \mathrm{EA}(69.47 \pm 1.18-102.69 \pm 2.87 \mu \mathrm{g} / \mathrm{mL})$ on CYP3A4 and CYP2D6. Based on the inhibitory potential of test samples, it can be concluded that $T$. arjuna and its standardized bioactive molecules could produce weak interaction potential when co-administered with conventional medicines.
\end{abstract}

Keywords: Drug metabolizing enzymes; Terminalia arjuna; Safety evaluation; Gallic acid; Ellagic acid; Drug interaction potential

\section{Introduction}

About $80 \%$ of people in developing countries still relay on traditional medicine their primary health care [1]. Elderly patients are using herbal drugs along with conventional prescription drugs due to their multiple illnesses [2]. When herbal drugs are co-administered with prescription drugs, there is an increasing risk of clinical treatment failures and adverse toxicity due to drug-herb interactions $[3,4]$. Terminalia arjuna (Roxb.) a deciduous tree of Combretaceae family, has been widely used in Indian system of medicine for cardiac ailments. Moreover, it is a vital component of common ayurvedic formulations available in India. It also possesses antioxidant, anti-inflammatory, antinociceptive and immunomodulatory activities [5-8] due to the presence of GA and EA along with other constituents.

CYP450 enzymes play a major role in the phase I oxidative metabolism of a wide range of pharmaceuticals [9]. Most of the pharmaceuticals are metabolized by or interact with only a small number of isoforms of CYP450. In this context, the most important forms are CYP2D6 and CYP3A4 [2,10,11]. Inhibition of CYP3A4 and/or CYP2D6 enzymes by herbal bioactive molecules may result in increased blood concentration of the target drug, which may lead to excessive accumulation and toxicity [12].

Various in vitro assays for measuring the activity and inhibition of CYP450 have been developed over the years [13]. HPLC-MS based assays are most commonly applied industrial standard method for CYP450 inhibition assessments. However, this method cannot be fit into high throughput format and may be difficult when huge number of samples needs to be tested. In this condition, fluorescence based CYP450 assays are much faster and more cost effective than HPLCMS assays [14,15]. In these assays, CYP450 oxidizes a pro-fluorescent molecule to a fluorescent product. This product can be directly measured using a fluorescent microplate reader. To our knowledge no data has been published on the interaction potential of T. arjuna plant extract or commercial product or its bioactive molecules on CYP450 mediated metabolism. Hence an attempt of systematic study has been taken to establish the safety issue through the interaction study with CYP450 including CYP3A4 and CYP2D6 isoforms.

\section{Materials and Methods}

\section{Chemicals}

All the chemicals and solvents for the research were of analytical grade. Vivid $^{\oplus}$ CYP450 Screening Kit and Vivid ${ }^{\circledR}$ Substrates were purchased from Invitrogen Drug Discovery Solutions, USA. Vivid ${ }^{\circledR}$ CYP3A4 Red (Cat. no. P2856) and Vivid ${ }^{\circledR}$ CYP2D6 Blue (Cat. no. P2972). HPLC grade Methanol, acetonitrile and acetic acid procured from Merck (Mumbai, India) were used. GA and EA were purchased from Sigma Chemical Co, St Louis, MO, USA. 96 well black flat bottom polystyrene not treated microplate was obtained from Corning (Costar \#3915, USA). Ketoconazole and quinidine was obtained as a gift sample from M/s Micro Labs Pvt. Ltd, Hosur, Tamil Nadu, India and M/s Trigenesis Life Sciences Pvt. Ltd, Bangalore, Karnataka, India respectively.

\section{Plant material and preparation of test samples}

T. arjuna, belonging to the family Combretaceae, holds a reputed position in both Ayurvedic and Yunani system of medicine. The bark of T. arjuna was collected from the central part of India (Madhya Pradesh)

*Corresponding author: Dr. TM Vijayakumar, Assistant Professor, Department of Pharmacy Practice, SRM University, Kattankulathur-603 203, Kancheepuram District, Tamil Nadu, India, Tel: +914427455818/+914447432300; Fax: +914427456702; E-mail: vijaypractice@yahoo.com

\section{Received April 10, 2017; Accepted May 23, 2017; Published May 29, 2017}

Citation: Vijayakumar TM, llango K, Vasanth K, Kasthuri Bai N, Mohan Kumar R, et al. (2017) Inhibitory Potency of Selected Therapeutic Bioactive Molecules of Standardized Terminalia arjuna (Roxb.) Extract on CYP3A4 and CYP2D6: Exploring Possible Herb-Drug Interactions. Nat Prod Chem Res 5: 272. doi: 10.4172/2329-6836.1000270

Copyright: (c) 2017 Vijayakumar TM, et al. This is an open-access article distributed under the terms of the Creative Commons Attribution License, which permits unrestricted use, distribution, and reproduction in any medium, provided the original author and source are credited. 
Citation: Vijayakumar TM, llango K, Vasanth K, Kasthuri Bai N, Mohan Kumar R, et al. (2017) Inhibitory Potency of Selected Therapeutic Bioactive Molecules of Standardized Terminalia arjuna (Roxb.) Extract on CYP3A4 and CYP2D6: Exploring Possible Herb-Drug Interactions. Nat Prod Chem Res 5: 270. doi: 10.4172/2329-6836.1000270

Page 2 of 7

in the month of September 2013, and the authentication was done by the Prof. Jayaraman, Plant Anatomy Research Centre, west Tambaram, Chennai-600 045, Tamil Nadu. A Voucher specimen (IIISM/RES/ HER082) has been preserved in the Interdisciplinary Institute of Indian System of Medicine (IIISM) department, SRM University for future reference. Hydro alcoholic (70\%) extract of coarsely powdered bark was prepared by cold maceration method, the extract was filtered and concentrated under reduced pressure by Rotovac (Buchi Rotavapor ${ }^{\circ}$ R-210). The extract and the bio-marker compounds GA and EA were dissolved in ethanol and dimethyl sulfoxide (DMSO) for the experiments.

\section{Standardization of the plant extract by RP-HPLC}

Quantitative analysis of GA and EA were performed using Reversephase high performance liquid chromatography system (RP-HPLC). The HPLC system consisted of a water 600 (Milford, MA, USA) binary HPLC pump, a Prominence $-7725 i$ injection valve (USA) with a sample loop of $20 \mu \mathrm{L}$, a water $2489 \mathrm{UV}$-visible dual wavelength detector and the max-plot containing the peaks were obtained using Lab solutions software. A phenomenex-Luna (Torrance, CA, USA) $\mathrm{C}_{18}$ column (250 $\mathrm{mm} \times 4.6 \mathrm{~mm}, 5 \mu \mathrm{m}$ particle size) was used as stationary phase. The isocratic mobile phase consists of methanol: acetonitrile: $1 \%$ of acetic acid in water (40:15:45) was used with a flow rate of $1 \mathrm{~mL} / \mathrm{min}$ and the samples were injected using Hamilton Microliter (Switzerland) syringe into a $20 \mu \mathrm{L}$ injection loop, the reference standards GA and EA was detected at $280 \mathrm{~nm}$ and $254 \mathrm{~nm}$ respectively. $1 \mathrm{mg} / \mathrm{ml}$ of the reference standards were dissolved in methanol and this stock solution was subsequently diluted to prepare solutions with concentrations in the range of $10-100 \mu \mathrm{L} / \mathrm{mL}$ of GA and EA separately. Exactly weighed test samples $(1 \mathrm{mg} / \mathrm{mL})$ were sonicated with methanol for $30 \mathrm{~min}$ and filtered through Whatman NYL $0.45 \mu$ syringe filter. An aliquot of 20 $\mu \mathrm{L}$ of test solutions was injected into the HPLC system at an interval of $10 \mathrm{~min}$. the analysis was repeated for three times by comparing and interpolating the extract peak area with that of standards from the calibration curve.

\section{Preparation of rat liver microsomes}

Based on the method labeled by Tang et al. [16], Male wistar rats (200-250 g) were used for CYP450-CO Complex assay. The present study was approved by the Institutional Animal Ethical Committee (IAEC151/2015), SRM College of Pharmacy, SRM University. Briefly the rats were sacrificed by cervical dislocation method and abdomen was opened, $1.15 \%$ cold $\mathrm{KCl}$ solution was injected into the liver portal vein until the liver turned into khaki color. Filter paper was used to absorb water and the liver tissue was rinsed with the volume of cold $50 \mathrm{mM}$ Tris- $\mathrm{HCl}$ buffer $(50 \mathrm{mM}$ Tris, $50 \mathrm{mM} \mathrm{KCl}, 0.5 \mathrm{mM}$ EDTA, $\mathrm{pH}$ 7.5) in three times to make homogenates. The homogenates were centrifuged (Micro Centrifuge-5430R, Eppendorf AG, Hamburg, Germany) at $10000 \mathrm{rpm}$ for $30 \mathrm{mins}$ at $4^{\circ} \mathrm{C}$ to remove cell debris, nuclei, mitochondria. The supernatant was transferred to fresh centrifuge tubes and centrifuged at $10500 \mathrm{rpm}$ for $60 \mathrm{~min}$ at $4^{\circ} \mathrm{C}$. The resulting pellet was resuspended in storage $0.2 \mathrm{M}$ sucrose into cryovials. All processes were performed $0-4^{\circ} \mathrm{C}$. Fractions were immediately frozen at $-80^{\circ} \mathrm{C}$ and used within a week. Protein concentrations were determined by modified biuret method (Multiskan ${ }^{\mathrm{m}}$ GO Microplate Spectrophotometer, Thermo Scientific, Waltham, MA, USA) using bovine serum albumin as standard.

\section{CYP450-Carbon monoxide (CO) complex assay}

Inhibitory activity of T. arjuna was performed with rat liver microsomes in 96 well microplate, based on method described by Ponnusankar et al. [17]. The concentration of $\mathrm{P} 450$ was calculated using the formula;

$$
P 450(m M)=\frac{\triangle A P C-\triangle A P}{91}
$$

Where, $\Delta \mathrm{A}_{\mathrm{PC}}$ is the absorbance difference of the PC sample, and $\Delta \mathrm{A}_{\mathrm{P}}$ is the absorbance difference of the $\mathrm{P}$ sample. The percentage inhibition was calculated using the following formula.

$$
\% \text { Inhibition }=\frac{\text { Blank }- \text { Test }}{\text { Blank }} \times 100
$$

High throughput fluorometric assay of CYP3A4 and CYP2D6

High throughput screening (HTS) assays were performed in black 96well microplates. Fluorescence readings were obtained on BioTekFLx 800 fluorescence microplate reader (Bio Tek, US) using appropriate excitation/emission $(\lambda)$ wavelength. The assay was performed according to a protocol provided by Invitrogen Drug Discovery Solutions, USA. Respective positive controls were used for different isozymes; concentration of enzymes and substrate has been described in Table 1. All measurements were performed in triplicate. Product formation from the fluorogenic probes were determined from the fluorescence data at seven different concentrations of the inhibitors and test. $\mathrm{IC}_{50}$ values were determined by using the following formula.

$$
\% \text { Inhibition }=\left(1-\frac{R F U \text { in presence of test compound }}{R F U \text { in absence of test compound }}\right) \times 100
$$

Where, RFU is Relative fluorescence unit.

\section{Molecular docking analysis}

Mode of inhibition of GA and EA on these CYPs was further assessed by molecular docking analysis with the software Glide (Schrodinger Inc. USA). Glide is a ligand docking program for predicting proteinligand binding modes and ranking ligands via high-throughput virtual screening [18]. Structures were downloaded from PubChem using chemical names. The compounds energy was minimized with various conformers, tautomers and ionization states using OPLS 2005 force field in LigPrep module in Schrodinger. Crystal structure of CYP3A4 and CYP2D6 were obtained from protein databank (PDB ID: 2F9Q and $1 T Q N)$ respectively. Using protein preparation wizard in Schrodinger module, protein was prepared for docking. In this step protein was minimized, assigning bond order, creating disulfide bond and water residues were removed. Those interacting water molecules with residues with protein and heteroatom were kept for docking studies. Then protein was refined by assigning $\mathrm{H}$-bonds and minimization at OPLS 2005 force field. The glide grid was generated around the cocrystallized ligand heme compound in crystal structure of CYP3A4 and CYP2D6 using receptor grid generation module. Using extra precision (XP) docking algorithm of Glide module, GA and EA docked with CYP3A4 and CYP2D6.

\section{Statistical analysis}

All data are presented as mean values standard error mean (S.E.M) of three replicates if not stated otherwise. The results were subjected to one way analysis of variance (ANOVA) and Dunnett's multiple comparison test was performed by fixing the significance level at $\mathrm{P}<0.05$ and above. $\mathrm{IC}_{50}$ values (concentration required to cause a $50 \%$ inhibition in enzyme activity) were obtained using mean enzyme activity versus inhibitor concentration curves using GraphPad prism Version 5.01 (GraphPad Prism Software Inc., USA). 
Citation: Vijayakumar TM, llango K, Vasanth K, Kasthuri Bai N, Mohan Kumar R, et al. (2017) Inhibitory Potency of Selected Therapeutic Bioactive Molecules of Standardized Terminalia arjuna (Roxb.) Extract on CYP3A4 and CYP2D6: Exploring Possible Herb-Drug Interactions. Nat Prod Chem Res 5: 270. doi: 10.4172/2329-6836.1000270

Page 3 of 7

\section{Results}

\section{Standardization of $T$. arjuna extract and its bioactive molecules by RP-HPLC}

The concentration of bioactive markers, GA and EA was determined through RP-HPLC using the isocratic condition and eluted through a run time of $10 \mathrm{~min}$. The separation was performed with the mobile phase consisting of methanol, acetonitrile, $1 \%$ of acetic acid in water in the ratio of 40:15:45 with a flow rate of $1 \mathrm{~mL} / \mathrm{min}$. The peak of the GA present in the extract was identified by comparing the retention time (Rt) of the reference standard at $3.00 \mathrm{~min}$ with no interference at 280 $\mathrm{nm}$ and the peak of EA was identified at $5.5 \mathrm{~min}$ with no interference at $254 \mathrm{~nm}$. In both the cases good correlations were found between concentrations and the peak area, with the correlation coefficient of more than 0.99 . Figures 1 and 2 represents the chromatograms obtained from HPLC analyses of the extract, standard GA and EA. The content of GA and EA in the test sample was found to be $0.41 \%(\mathrm{w} / \mathrm{w})$ and $0.16 \%$ $(\mathrm{w} / \mathrm{w})$ respectively.

\section{Determination of CYP450 concentration and percentage inhibition through CYP450-CO Complex assay}

Cytochrome inhibition assay through cytochrome $\mathrm{P} 450-\mathrm{CO}$ complex method in presence of specific CYP inhibitor is the first approach to study the inhibitory potential. Concentration-dependent inhibition of CYP450 was shown that test sample and its constituents had much less potential to inhibit the CYP enzymes. The concentration of protein in isolated rat liver microsome was found to be $7.7 \mathrm{mg} / \mathrm{mL}$. Cytochrome P450-CO assay was used to assess the inhibitory potential of T. arjuna. Ethanol and DMSO solution of the extract, GA and EA indicated a concentration reliant inhibition of CYP450 (Figure 3A and 3B). The CYP450 concentration of the diluted liver microsome was calculated to be about $0.304 \mathrm{nmol} / \mathrm{mg}$ protein. Results shown that plant extract, GA and EA exhibited significantly less inhibition than Ketoconazole and Quinidine (Figure 3C). Two different solvents were used to evaluate the CYP450 inhibition, the results revealed minor variation; this confirms that the solvent effect was minimized. T. arjuna extract $(31.02 \pm 2.24 \%)$, GA $(28.14 \pm 2.45 \%)$ and EA $(18.00 \pm 1.16 \%)$ in DMSO showed slight

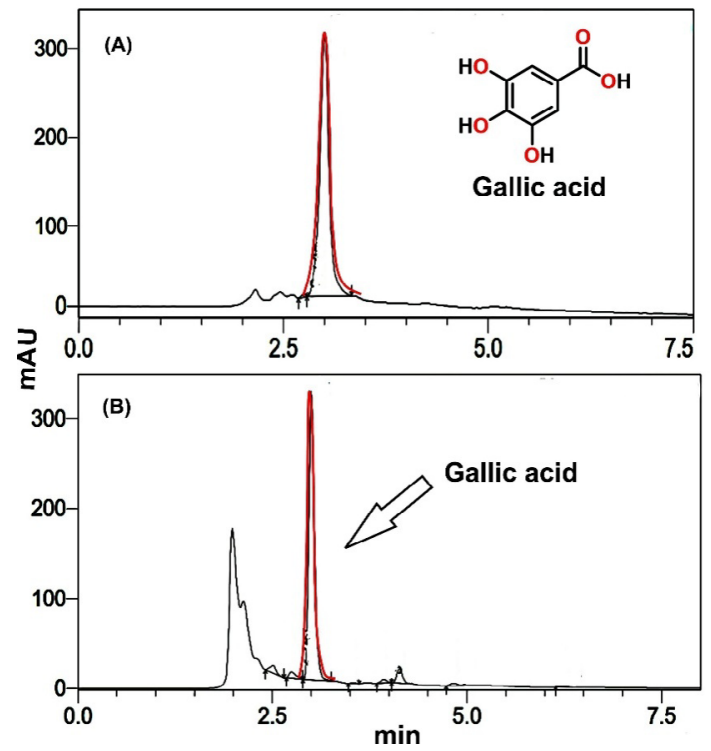

Figure 1: A. HPLC chromatogram of standard GA and B. HPLC chromatogram of $T$. arjuna hydro alcoholic extract.

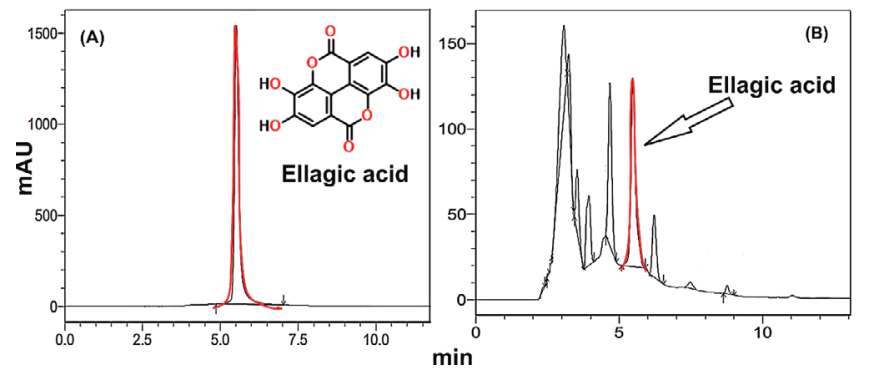

Figure 2: A. HPLC chromatogram of standard EA and B. HPLC chromatogram of $T$. arjuna hydro alcoholic extract.

highest percentage of inhibition. An organic solvent such as DMSO and ethanol strongly affect the metabolism at 5\% concentration level [9]. The variation in inhibition was detected with all the DMSO solubilized extract, when compared with the ethanol solubilized extracts. But, to confirm the possible inhibition by the test samples, suitable solvent controls were used in the study and the percentage inhibition was calculated after counteracting the solvent effect. So, it was confirmed that the concentrations of DMSO and ethanol used in this study did not interfere in the CYP450 interactions.

\section{Determination of $\mathrm{IC}_{50}$ by high throughput fluorometric assay}

Fluorometric assays helps to ensure the drug interaction potential of test compound through the important CYP isoforms (CYP3A4, 2D6). Inhibitor potency was quantified by determination of $\mathrm{IC}_{50}$ values. T. arjuna extract, GA and EA were assayed between concentrations ranging from 1.5 to $25 \mu \mathrm{g} / \mathrm{mL}$. All tests were assayed in triplicate, and $\mathrm{IC}_{50}$ values were calculated using endpoint mode (Table 2). Concentration reliant percentage inhibitions of the test compound on CYP3A4 and CYP2D6 were observed (Figure 4). The study results shown that the $T$. arjuna extract and the constituents had less inhibition potential on the CYP3A4 and CYP2D6 compared to their respective positive controls. $\mathrm{IC}_{50}$ values for positive inhibitors were in good agreement to previous studies [19]. T. arjuna in DMSO exhibited a better activity against CYP3A4 and CYP2D6 but IC $_{50}$ value $(48.06 \pm 1.14-57.89 \pm 2.15 \mu \mathrm{g} /$ $\mathrm{mL}$ ) seemed high when compared to the respective positive controls $(07.20 \pm 0.56-06.28 \pm 1.76 \mu \mathrm{g} / \mathrm{mL})$. Results indicated that the test extract have higher inhibition potential comparing to their individual bioactive compounds. The higher enzyme inhibition potential by the extracts may be related to the synergistic effects due to presence of other constituents in the extract.

\section{Docking studies of active constituents and controls within CYP3A4 and CYP2D6 molecule active site}

Molecular docking analysis on the interactions of GA and EA to CYP3A4 and CYP2D6 were performed to confirm the drug binding conformation. GA and EA had docking score of -8.55 and -8.36 $\mathrm{kcal} / \mathrm{mol}$ with CYP3A4 respectively, which is lower than that of the Ketoconazole-CYP3A4 complex $(-9.10 \mathrm{kcal} / \mathrm{mol})$ (Figure 5E). GA has been interacted via van der Waals with PHE 302, ASN 451, GLY 444 (Figure 5A) and EA was interacted with THR 310, ALA 305, PRO 434 and ALA 370 respectively with CYP3A4 (Figure 5B). With CYP2D6, GA and EA had docking score of -7.44 and $-8.17 \mathrm{kcal} / \mathrm{mol}$ respectively, which is less than that of the Quinidine-CYP2D6 complex (-10.48 $\mathrm{kcal} / \mathrm{mol}$ ) (Figure $5 \mathrm{~F}$ ). GA also interacted via van der Waals with PRO 435, SER 437, HIS 376, MET 374, ARG 101 (Figure 5C) and EA was interacted with SER 473, PRO 435, MET 374, HIS 376, ARG 441, and ARG 101 AND ALA 305 respectively with CYP2D6 (Figure 5D). It was 
Citation: Vijayakumar TM, Ilango K, Vasanth K, Kasthuri Bai N, Mohan Kumar R, et al. (2017) Inhibitory Potency of Selected Therapeutic Bioactive Molecules of Standardized Terminalia arjuna (Roxb.) Extract on CYP3A4 and CYP2D6: Exploring Possible Herb-Drug Interactions. Nat Prod Chem Res 5: 270. doi: 10.4172/2329-6836.1000270
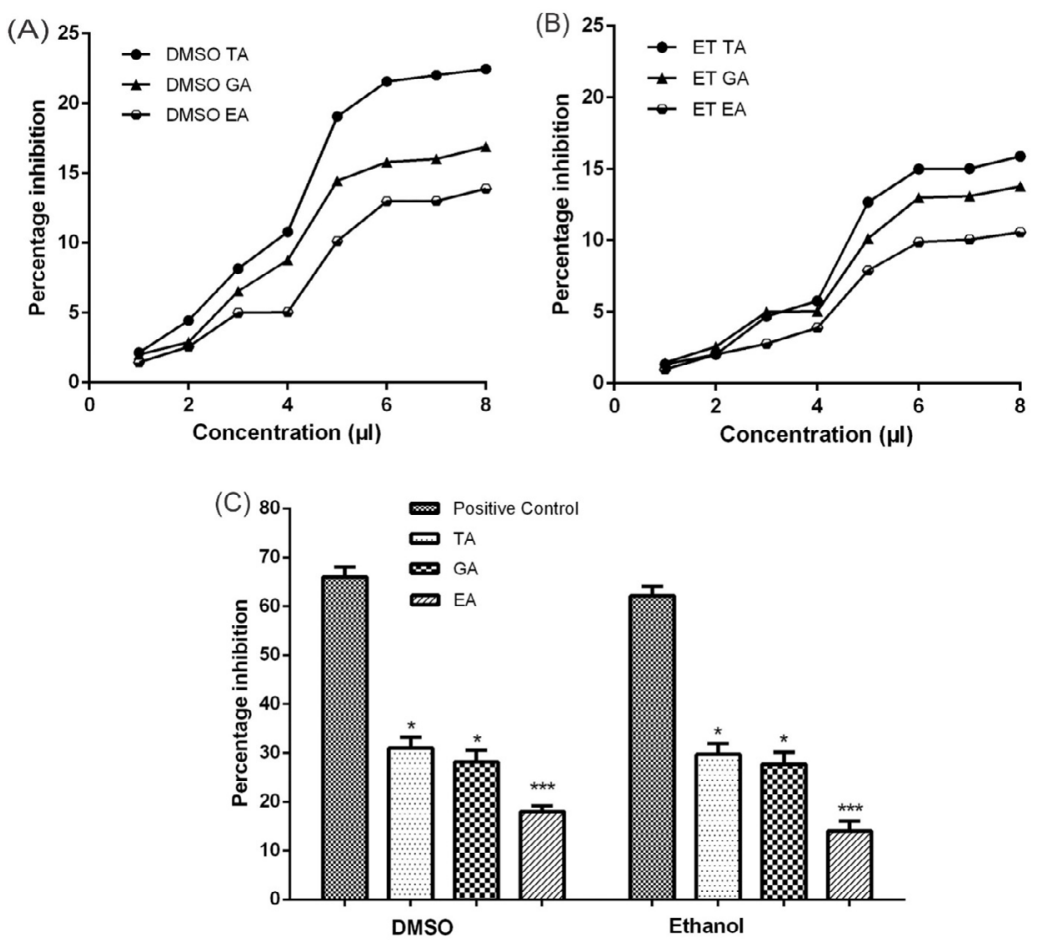

Figure 3: A and B. Concentration dependent inhibition of the T. arjuna extract, GA and EA in DMSO and Ethanol. C: Percentage inhibition of T. arjuna, GA and EA versus positive control Ketoconazole; Values are expressed in mean $\pm \mathrm{SEM} ; n=3$; ANOVA followed by Dunnett's multiple comparison Test. Level of significance * $p<0.05$, ${ }^{* *} p<0.01$ and ${ }^{* * *} p<0.001$.
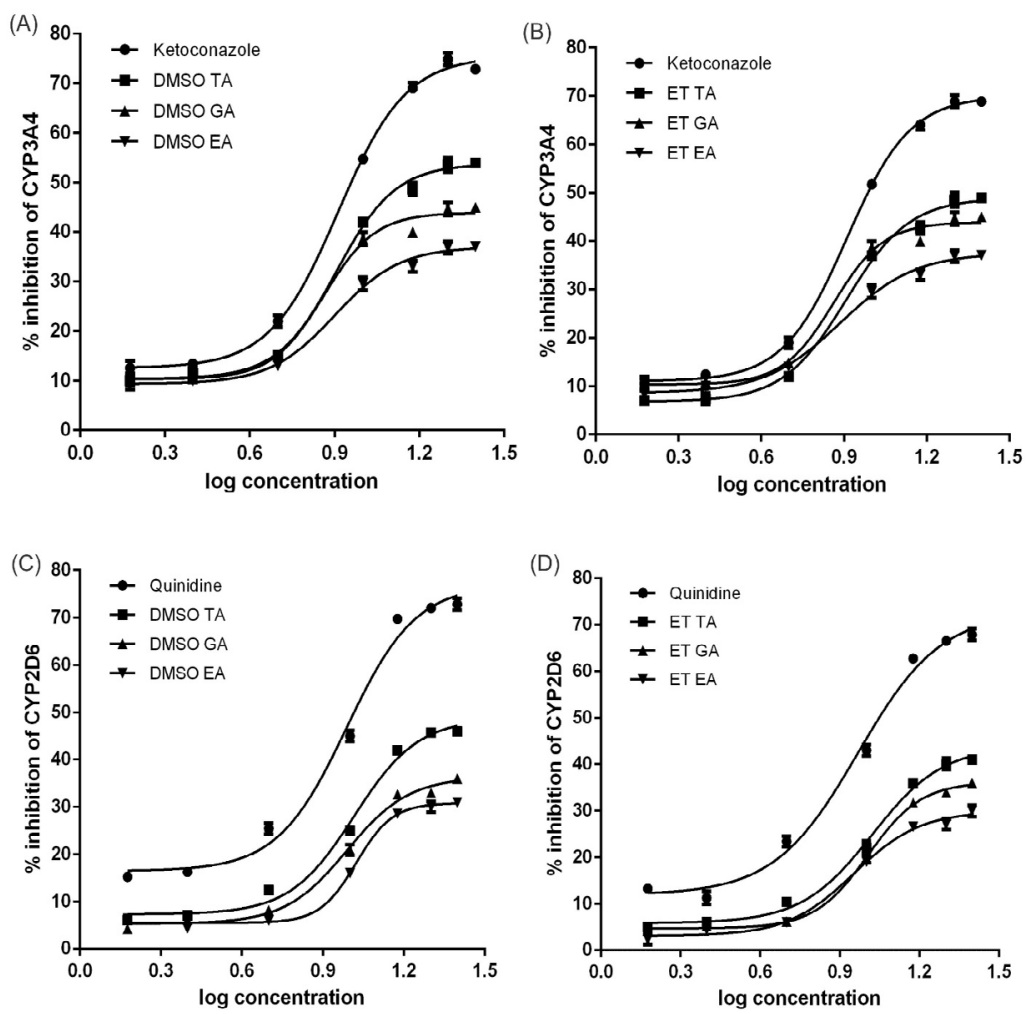

Figure 4: Concentration dependent inhibitory effect of T. arjuna extract and bioactive compounds on CYP3A4 in DMSO (A) and Ethanol (B) versus Ketoconazole, CYP2D6 in DMSO (C) and Ethanol (D) versus Quinidine. 
Citation: Vijayakumar TM, Ilango K, Vasanth K, Kasthuri Bai N, Mohan Kumar R, et al. (2017) Inhibitory Potency of Selected Therapeutic Bioactive Molecules of Standardized Terminalia arjuna (Roxb.) Extract on CYP3A4 and CYP2D6: Exploring Possible Herb-Drug Interactions. Nat Prod Chem Res 5: 270. doi: 10.4172/2329-6836.1000270

Page 5 of 7

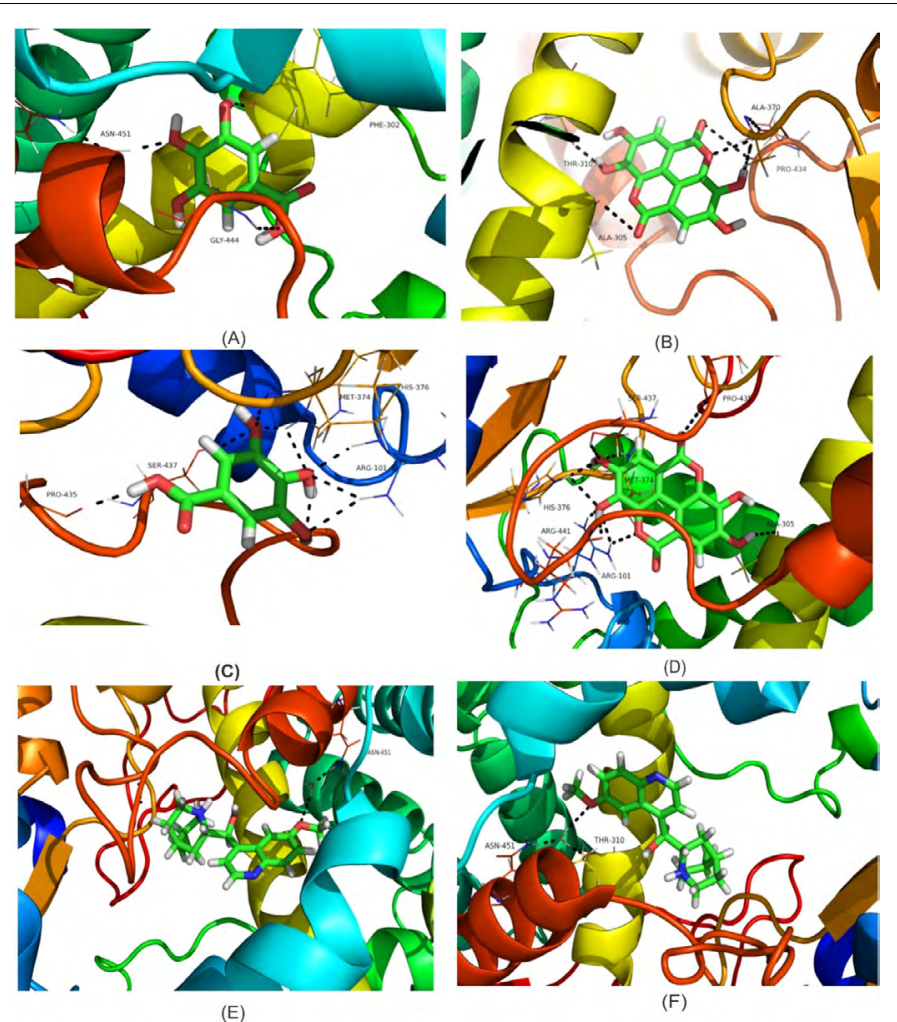

Figure 5: Predicted binding sites poses of $G A(A)$ and $E A(B)$ within the active site of CYP3A4 molecule by docking, GA (C) and EA (D) within the active site of CYP2D6, Ketoconazole (E) within the active site of CYP3A4 and Quinidine (F) within the active site of CYP2D6.

\begin{tabular}{|c|c|c|}
\hline $\begin{array}{c}\text { Experimental conditions/ CYP } \\
\text { isoforms }\end{array}$ & CYP3A4 & CYP2D6 \\
\hline Substrate concentration & $3 \mu \mathrm{M}$ & $10 \mu \mathrm{M}$ \\
\hline Concentration of fluorescent standard & $100 \mu \mathrm{M}$ & $100 \mu \mathrm{M}$ \\
\hline Master Pre-mix screening concentration & $5 \mathrm{nM}$ & $10 \mathrm{nM}$ \\
\hline Positive inhibitor & Ketoconazole & Quinidine \\
\hline Concentration of inhibitor & $10 \mu \mathrm{M}$ & $1 \mu \mathrm{M}$ \\
\hline Incubation time & $30 \mathrm{~min}$ & $30 \mathrm{~min}$ \\
\hline Excitation wavelength & $530 \mathrm{~nm}$ & $360 \mathrm{~nm}$ \\
\hline Emission wavelength & $590 \mathrm{~nm}$ & $460 \mathrm{~nm}$ \\
\hline
\end{tabular}

Master Pre-mix (recombinant human Cytochrome P450, glucose-6-phosphate and glucose-6-phosphate dehydrogenase)

Table 1: High throughput fluorescence inhibition assay experimental conditions.

\begin{tabular}{|c|c|c|c|}
\hline \multirow{2}{*}{ Test sample } & Solvent & $\begin{array}{c}\mathbf{I C}_{50}(\boldsymbol{\mu g} / \mathbf{m l}) \\
\text { (CYP3A4) }\end{array}$ & $\begin{array}{c}\mathbf{I C}_{50}(\boldsymbol{\mu g} / \mathbf{m l}) \\
\text { (CYP2D6) }\end{array}$ \\
\hline \multirow{2}{*}{ T. arjuna } & DMSO & $48.06 \pm 1.14^{\mathrm{a}}$ & $53.66 \pm 1.36^{\mathrm{b}}$ \\
\cline { 2 - 4 } & Ethanol & $53.36 \pm 2.45^{\mathrm{a}}$ & $57.89 \pm 2.15^{\mathrm{b}}$ \\
\hline \multirow{2}{*}{ GA } & DMSO & $66.54 \pm 1.04^{\mathrm{a}}$ & $78.46 \pm 1.32^{\mathrm{b}}$ \\
\cline { 2 - 4 } & Ethanol & $72.13 \pm 2.67^{\mathrm{a}}$ & $83.84 \pm 1.06^{\mathrm{b}}$ \\
\hline \multirow{2}{*}{ EA } & DMSO & $69.47 \pm 1.18^{\mathrm{a}}$ & $97.34 \pm 1.55^{\mathrm{b}}$ \\
\cline { 2 - 4 } & Ethanol & $74.32 \pm 2.08^{\mathrm{a}}$ & $102.69 \pm 2.87^{\mathrm{b}}$ \\
\hline \multirow{2}{*}{ Ketoconazole } & DMSO & $07.20 \pm 0.56$ & - \\
\cline { 2 - 4 } & Ethanol & $07.74 \pm 1.32$ & - \\
\hline \multirow{2}{*}{ Quinidine } & DMSO & - & $05.84 \pm 0.68$ \\
\cline { 2 - 4 } & Ethanol & - & $06.28 \pm 1.76$ \\
\hline
\end{tabular}

Values are expressed in mean $\pm \mathrm{SEM},{ }^{\mathrm{a}} \mathrm{P}<0.001,{ }^{\mathrm{b}} \mathrm{P}<0.001$ versus positive contro ketoconazole and quinidine respectively. Abbreviations: DMSO: dimethyl sulfoxide; GA: gallic acid; EA: ellagic acid

Table 2: $\mathrm{IC}_{50}(\mu \mathrm{g} / \mathrm{mL})$ value of $T$. arjuna extract, $\mathrm{GA}$ and EA on CYP3A4 and CYP2D6. found that all the ligands were involved in hydrogen bonding with the binding site residues of CYP450. Overall molecular docking studies concluded that GA and EA have less glide score and glide Energy with both CYP3A4 and CYP2D6 when compared to respective positive controls energy and score. Molecular docking results indicated that GA and EA has weaker inhibition activity of the CYP3A4 and CYP2D6. Therefore the in-silico prediction was in agreement with the results obtained from the CYP3A4 and CYP2D6 inhibition assays.

\section{Discussion}

Cytochrome P450 enzymes have been of particular interest in the field of drug discovery for numerous reasons including the involvement of these enzymes in the metabolism of over $95 \%$ of the drugs on the market and the potential of drug-drug interaction through metabolism. Drug interactions are not limited to synthetic drugs, herbal products may also act as triggers for changes in the CYP activity; for example CYPmodulating effects are described for echinacea, grape fruit juice or St. John's Wort [20,21]. Administration of herbs and allopathic medicine may increase the chance of herb-drug interaction, which results in an increase of the plasma drug concentration and produces toxicity. To avoid or minimize toxic drug-herb interactions, it is important to identify drugs that can interact with herbs using proper in vitro, in silico and in vivo models in the early stages of drug development. Through this study an approach was made to evaluate the interaction potential of T. arjuna with conventional pharmaceuticals.

A number of in vitro systems can be used to investigate herb-CYP interactions (e.g., liver microsomes, precision-cut liver slices, cultured hepatocytes, and cDNA-expressed enzymes) [22-26]. We have adopted a high-throughput approach to screen the inhibitory effect of test substances on two major drug-metabolizing CYP enzymes. High 
Citation: Vijayakumar TM, llango K, Vasanth K, Kasthuri Bai N, Mohan Kumar R, et al. (2017) Inhibitory Potency of Selected Therapeutic Bioactive Molecules of Standardized Terminalia arjuna (Roxb.) Extract on CYP3A4 and CYP2D6: Exploring Possible Herb-Drug Interactions. Nat Prod Chem Res 5: 270. doi: 10.4172/2329-6836.1000270

Page 6 of 7

throughput screening assays may represent a useful strategy for the study of herb-CYP interactions. They are capable of handling the great number of herbal constituents, and have the ability to provide in vitro inhibition data as a criterion for monitoring herb-drug metabolic interactions involving human CYP enzymes. Cytochrome inhibition assay was performed through cytochrome P450-CO complex method in presence of specific CYP inhibitor. Concentration-dependent inhibition of CYP450 was shown that test sample and its constituents have much less potential to inhibit the CYP enzymes.

In silico approaches represent a useful tool for the study of herbCYP interactions as demonstrated by our study. Our established pharmacophore model could readily distinguish the most potent inhibitor if CYP3A4 and 2D6. Thus, this model could be used as a high throughput-screening tool to identify natural constituents of herbal preparations that inhibit CYP3A4 and 2D6, before undertaking in vitro determinations [27]. This will help avoid co-administration of drugs that are extensively metabolized by CYP3A4 and 2D6 with herbal products that showed potent inhibitory effects on these enzymes.

However, the results shown that the T. arjuna extract and its bioactive molecules studied in this study inhibits the CYP3A4 and CYP2D6 with an $\mathrm{IC}_{50}$ values of $>0.103 \mathrm{mg} / \mathrm{mL}$. In contrast, a relatively strong inhibition was observed with known inhibitors such as quinidine and ketoconazole with an $\mathrm{IC}_{50}$ value of $<0.0078 \mathrm{mg} / \mathrm{mL}$. From these findings, it was observed that the test substances have very weak inhibitory potential on CYP450 and its important isoforms (CYP3A4, CYP2D6). The order of inhibitory potential of the test substances through in vitro results were identified as TA $>$ GA $>$ EA. The extract and its bioactive molecules tested in the present study had very weak interaction with other drug biotransformation but the observed inhibitory effect on metabolizing enzymes can be highly variable as the constituent content can differ with plant species, source, environment, and processing and storage conditions.

However, herb-drug interactions are difficult to characterize and resolve, because of the lack of comprehensive federal regulations regarding safety, efficacy, and manufacturing standards for herbal medicines. It has been recommended that herbs are appropriately labelled to alert consumers to possible interactions with other concomitantly used drugs and to recommend a consultation with their general practitioners, pharmacists, and/or other medical carers. It is time to consider herbs not as alternative medicine based on tradition and experience, but as Phytotherapy, an integrated part of medical treatment [28]. Regulations with regard to safety (e.g., herb-drug interactions), quality and efficacy of herbs would be highly desirable. Thus, monitoring of adverse events when herbal medicines are co-administered with drugs can be systematically carried out and potential herb-drug interactions be identified. This would enable more accurate product labelling and a body of useful information on potential herb-drug interactions to medical professionals.

\section{Conclusion}

In conclusion, our study highlighted the inhibitory potency of T. arjuna and its standardized therapeutic bioactive compounds on CYP450 and its significant isoforms. Even though results indicated that test extracts and bioactive molecules had weak interaction potential, time interval should be considered based on elimination half-life of therapeutic drugs when $T$. arjuna containing herbal formulation administered. In vitro and in silico evaluation of Herb-CYP450 interactions can be incorporated to find out the interaction inducing molecules in the early stages of drug development.

\section{Acknowledgements}

We would like to thank Department of Science and Technology, Government of India (Grant number: VI-D\&P/372/10-11/TDT) for their financial assistance and support. We thank Dr. M. Muthu Tamizh (CCRS, Chennai) and Dr. RC Satish Kumar (IIISM, SRM University) for helpful discussion.

\section{Conflict of Interest}

The authors declare that there are no conflicts of interest.

\section{References}

1. Verma S, Singh SP (2008) Current and future status of herbal medicines. Vet World 1: 347-350.

2. Schroder-Aasen T, Molden G, Nilsen OG (2012) In vitro inhibition of CYP3A4 by the multiherbal commercial product Sambucus Force and its main constituents Echinacea purpurea and Sambucus nigra. Phytotherapy research 26: 1606-1613.

3. Usia T, Watabe T, Kadota S, Tezuka Y (2005) Cytochrome P450 2D6 (CYP2D6) inhibitory constituents of Catharanthus roseus. Biological \& pharmaceutical bulletin 28: 1021-1024

4. Mukherjee PK, Ponnusankar S, Pandit S, Hazam PK, Ahmmed M, et al. (2011) Botanicals as medicinal food and their effects on drug metabolizing enzymes. Food and chemical toxicology 49: 3142-3153.

5. Gupta R, Singhal S, Goyle A, Sharma VN (2001) Antioxidant and hypocholesterolaemic effects of Terminalia arjuna tree-bark powder: a randomised placebo-controlled trial. The Journal of the Association of Physicians of India 49: 231-235.

6. Dwivedi S, Agarwal MP (1994) Antianginal and cardioprotective effects of Terminalia arjuna, an indigenous drug, in coronary artery disease. The Journal of the Association of Physicians of India 42: 287-289.

7. Dwivedi S, Jauhari R (1997) Beneficial effects of Terminalia arjuna in coronary artery disease. Indian heart journal 49: 507-510.

8. Bharani A, Ganguly A, Bhargava KD (1995) Salutary effect of Terminalia Arjuna in patients with severe refractory heart failure. International journal of cardiology 49: 191-199.

9. Pandit S, Ponnusankar S, Bandyopadhyay A, Ota S, Mukherjee PK (2011) Exploring the possible metabolism mediated interaction of Glycyrrhiza glabra extract with CYP3A4 and CYP2D6. Phytotherapy research 25: 1429-1434.

10. llango K, Vijayakumar TM, Agrawal A, Dubey GP (2013) Pharmacogenetic Comprised Prospect on Cytochrome P450 Facilitated Drug Interactions. Journal of Bionanoscience 7: 127-139.

11. Zhou SF, Xue CC, Yu XQ, Li C, Wang G (2007) Clinically important drug interactions potentially involving mechanism-based inhibition of cytochrome P450 3A4 and the role of therapeutic drug monitoring. Therapeutic drug monitoring 29: 687-710.

12. Subehan S, Usia T, Iwata H, Kadota S, Tezuka Y (2006) Mechanism-based inhibition of CYP3A4 and CYP2D6 by Indonesian medicinal plants. Journal of ethnopharmacology 105: 449-455.

13. Fowler S, Zhang H (2008) In vitro evaluation of reversible and irreversible cytochrome P450 inhibition: current status on methodologies and their utility for predicting drug-drug interactions. AAPS journal 10: 410-424.

14. Schaeffner I, Petters J, Aurich H, Frohberg P, Christ B (2005) A microtiterplatebased screening assay to assess diverse effects on cytochrome P450 enzyme activities in primary rat hepatocytes by various compounds. Assay and drug development technologies 3: 27-38.

15. Yamamoto T, Suzuki A, Kohno Y (2002) Application of microtiter plate assay to evaluate inhibitory effects of various compounds on nine cytochrome P450 isoforms and to estimate their inhibition patterns. Drug metabolism and pharmacokinetics 17: 437-448.

16. Tang JC, Yang $H$, Song $X Y$, Song $X H$, Yan SL, et al (2009) Inhibition of cytochrome $\mathrm{P} 450$ enzymes by rhein in rat liver microsomes. Phytotherapy research 23: 159-164.

17. Ponnusankar S, Pandit S, Babu R, Bandyopadhyay A, Mukherjee PK (2011) Cytochrome P450 inhibitory potential of Triphala--a Rasayana from Ayurveda. Journal of ethnopharmacology 133: 120-125. 
Citation: Vijayakumar TM, llango K, Vasanth K, Kasthuri Bai N, Mohan Kumar R, et al. (2017) Inhibitory Potency of Selected Therapeutic Bioactive Molecules of Standardized Terminalia arjuna (Roxb.) Extract on CYP3A4 and CYP2D6: Exploring Possible Herb-Drug Interactions. Nat Prod Chem Res 5: 270. doi: 10.4172/2329-6836.1000270

Page 7 of 7

18. Repasky MP, Shelley M, Friesner RA (2007) Flexible ligand docking with Glide. Current protocols in bioinformatics 8: 12

19. Stresser DM, Blanchard AP, Turner SD, Erve JC, Dandeneau AA, et al. (2000) Substrate-dependent modulation of CYP3A4 catalytic activity: analysis of 27 test compounds with four fluorometric substrates. Drug metabolism and disposition 28: 1440-1448.

20. Modarai M, Yang M, Suter A, Kortenkamp A, Heinrich M (2010) Metabolomic profiling of liquid Echinacea medicinal products with in vitro inhibitory effects on cytochrome P450 3A4 (CYP3A4). Planta medica 76: 378-385

21. Heinrich M, Modarai M, Kortenkamp A (2008) Herbal extracts used for uppe respiratory tract infections: are there clinically relevant interactions with the cytochrome P450 enzyme system? Planta medica 74: 657-660.

22. Lu P, Schrag ML, Slaughter DE, Raab CE, Shou M, et al. (2003) Mechanismbased inhibition of human liver microsomal cytochrome P450 1A2 by zileuton, a 5-lipoxygenase inhibitor. Drug metabolism and disposition: the biological fate of chemicals 31: 1352-1360.
23. Sevior DK, Hokkanen J, Tolonen A, Abass K, Tursas L, et al. (2010) Rapid screening of commercially available herbal products for the inhibition of major human hepatic cytochrome P450 enzymes using the $\mathrm{N}$-in-one cocktail. Xenobiotica 40: 245-254.

24. Gurley BJ, Swain A, Hubbard MA, Williams DK, Barone G, et al. (2008) Clinica assessment of CYP2D6-mediated herb-drug interactions in humans: Effects of milk thistle, black cohosh, goldenseal, kava kava, St. John's wort, and Echinacea. Molecular Nutrition \& Food Research 52: 755-763.

25. Komoroski BJ, Zhang S, Cai H, Hutzler JM, Frye R, et al. (2004) Induction and inhibition of cytochromes P450 by the St. John's wort constituent hyperforin in human hepatocyte cultures. Drug metabolism and disposition 32: 512-518.

26. Zou L, Harkey MR, Henderson GL (2002) Effects of herbal components on cDNA-expressed cytochrome P450 enzyme catalytic activity. Life sciences 71 1579-1589.

27. Zhou S, Gao Y, Jiang W, Huang M, Xu A, et al. (2003) Interactions of herbs with cytochrome P450. Drug metabolism reviews 35: 35-98.

28. Qiu J (2007) China plans to modernize traditional medicine. Nature 446: 590-591. 